\title{
STRUCTURE OF THE OTOLITHIC LAYERS ON THE MACULAE SACCULI AND UTRICULI IN THE GUINEA PIG
}

\author{
Kozo Watanuki, Kazutomo Kawamoto, and Shuichi Katagiri
}

\section{INTRODUCTION}

The macula sacculi belongs to the pars inferior of the labyrinth, while the macula utriculi to the pars superior. Although the saccule may have something to do with sound perception, both otolithic organs are generally considered to be gravity and vibration receptors. Both maculae are similar in structure. The otolithic layer, however, shows different structures in different regions of the macula. In the present study, the otolithic layer was studied with a special reference to the relationship between the otolithic layer and the sensory epithelium.

\section{MATERIAL AND METHODS}

Fifty young guinea pigs, each weighing about $300 \mathrm{~g}$, were used in this study. After removal of the temporal bone, the stapes was lifted with the surrounding bony wall of the vestibule. Then the temporal bone was fixed in $3.0 \%$ buffered glutaldehyde $(2.0 \mathrm{M}$ Phosphate buffer $\mathrm{pH}$ 7.4). Epon sections $(2-5 \mu)$ were stained with toluidine blue and studied under the light microscope. For the surface study of the otolithic membranes and the otolithic layers, the temporal bone was fixed in $2.0 \%$ buffered osmiumtetroxide (2.0 M Phosphate buffer $\mathrm{pH}$ 7.4). The entire otolithic membrane with the otoliths was separated from the sensory epithelium and sucked by a small glass-pipette in one piece. The otolithic membranes with the otoliths were placed on a slide-glass and were dried in the air. Under oblique or vertical illumination, the entire upper and lower surfaces of the otolithic membrane and the otolithic layer were studied with an ordinary light microscope. For studying the otolithic membranes, some specimens were decalcified by EDTA and stained with hematoxylin.

\section{OBSERVATIONS}

The maculae utriculi and sacculi were mainly vertical to each other. The macula utriculi were located in the recessus utriculi, while the macula sacculi were in the recessus sphericus of the vestibule. Generally the macula utriculi lay in a horizontal plane, while the macula sacculi in a vertical plane. The otolithic membranes and the otolithic layers covered the sensory epithelia of both maculae with sufficient margins. The otolithic mem-

From the Department of Otolaryngology, Tohoku University School of Medicine, Sendai 

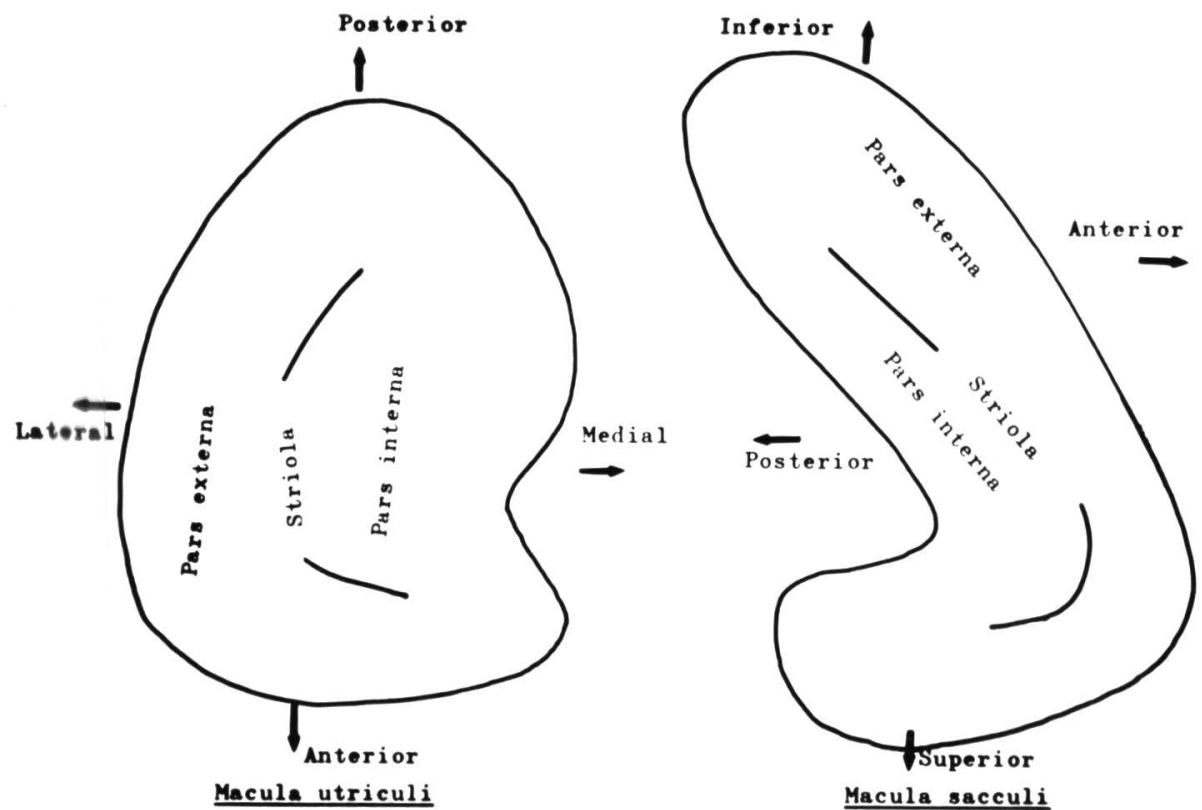

Fig. 1. Orientation of the location of the macula sacculi and utriculi (Lindeman $1969^{9}$ ). The striola divides each macula into two areas (the pars interna and the pars externa) with opposite morphological polarization of the sensory cells.

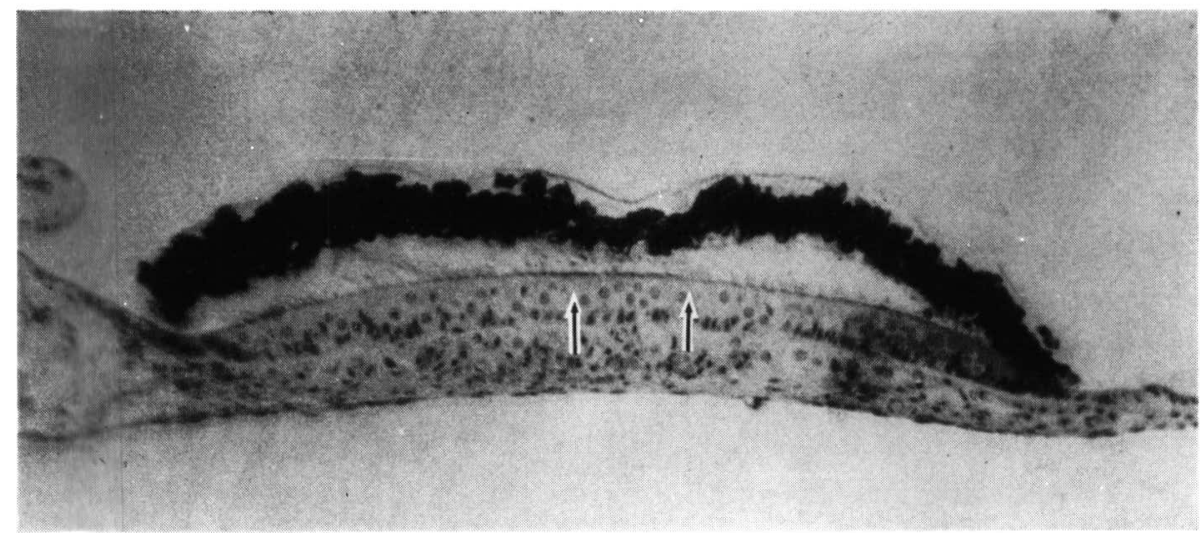

Fig. 2. Medial-lateral section of the posterior part of the macula utriculi. The striola is indicated between the two arrows. $100 \times$.

branes and the otolithic layers of both maculae were principally similar in structure. The otoliths were embedded in the upper layer of the otolithic membranes. The kinociliae and the stereociliae of the sensory cells were in direct contact with the lower layer of the otolithic membrane. The otolithic membrane consisted of a gelatin-like substance containing the reticular structure. This reticular structure was more distinct and thicker in the striola than in the other peripheral regions (Fig. 6). The otoliths were elongated hexagonal shapes with blunted corners. They vary considerably in size in different regions of the macula. In general, the otoliths were larger in the lower portion of the otolithic layer and smaller toward the upper surface of it. In the striola, in the narrow margi- 


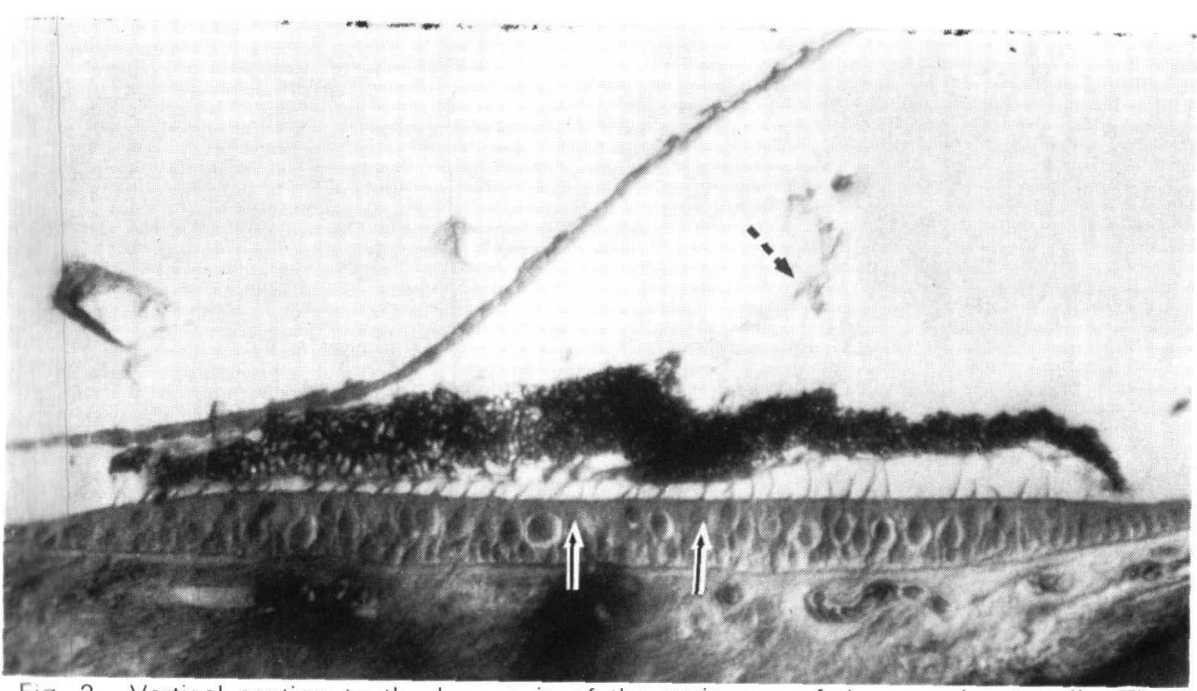

Fig. 3. Vertical section to the long-axis of the main part of the macula sacculi. The striola is indicated between the two thick arrows. A broken arrow indicates a very thin membrane structure of unknown nature in contact with the surface of the otoltihic layer. $100 \times$.

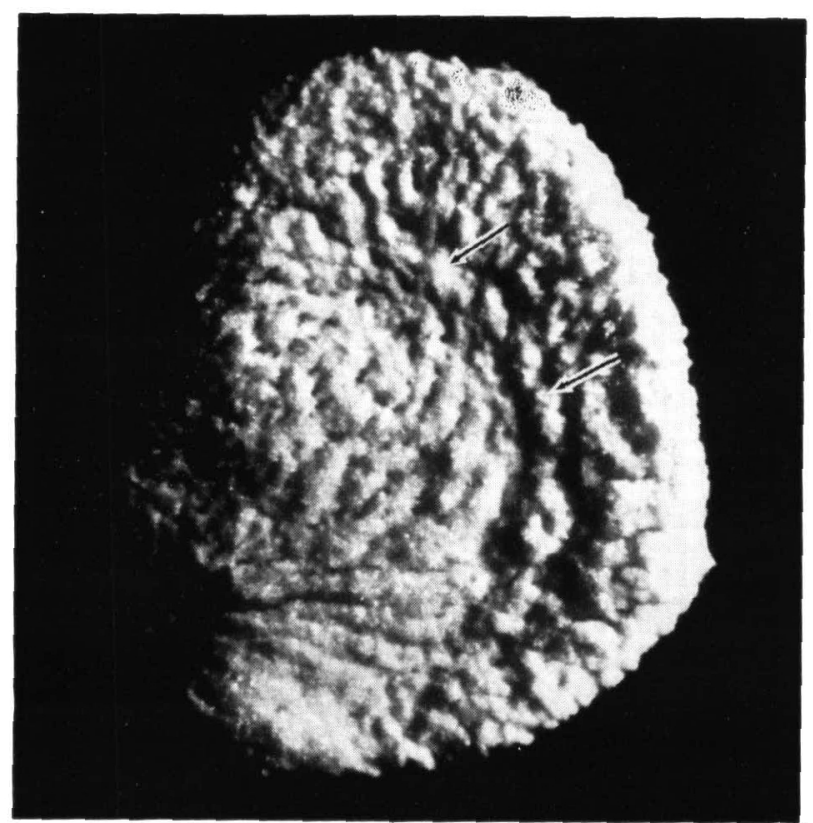

Fig. 4. Surface view of the otolithic layer in the macula utriculi with side illumination. In the groove of the striola, a narrow slight elevation or the otolithic layer is seen (Arrow). $50 \times$.

nal zones, in the medial indented portion of the macula utriculi as well as in the superiorposterior indented portion of the macula sacculi, the otoliths were very small and varied less in size. Often large otoliths were found to be disributed regularly with the long-axis perpendicular to the sensory epithelium. The marginal zone of the otolithic layer was narrower in the lateral side than in the medial side of the macula utriculi, while it was narrower in the anterior-inferior side than in the posterior-superior side of the macula sacculi. 


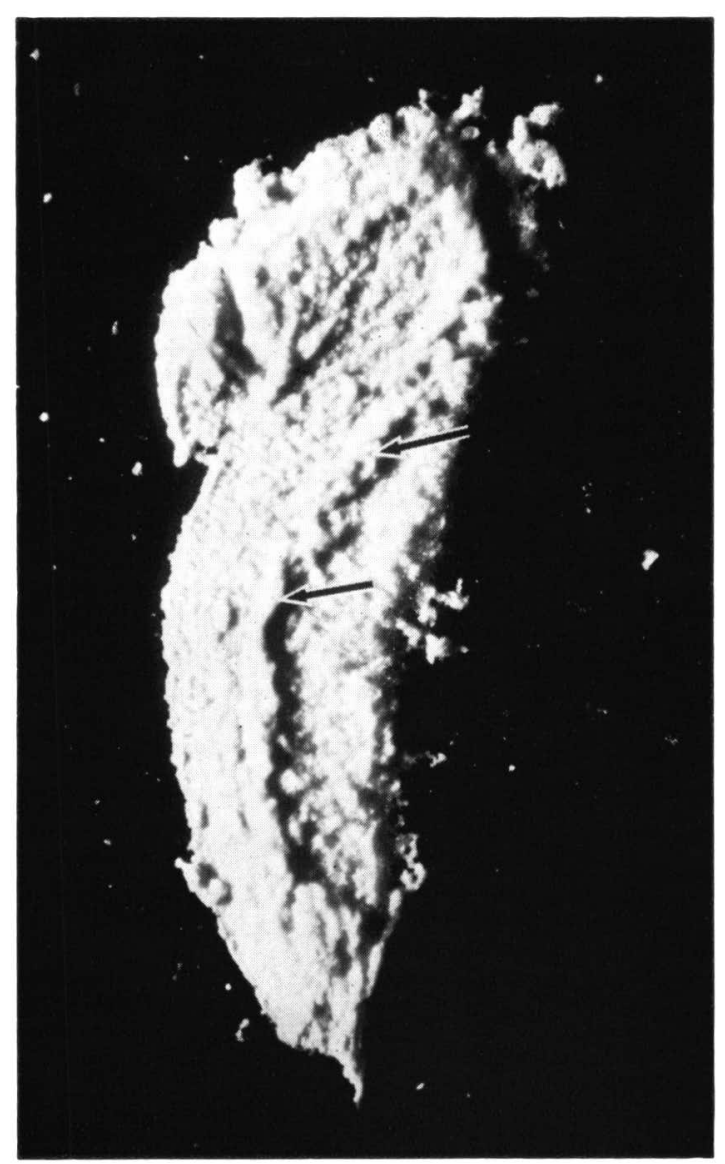

Fig. 5. Surface view of the otolithic layer in the macula sacculi with side illumination. In the striola region, a range of otolithic hills is seen (Arrow). $50 \times$.

1) The otolithic membrane and the otoliths of the macula utriculi:

The main part of the macula utriculi lay in a horisontal plane with the head in an up-right position. The anterior one-fourth of the macula, however, was elevated at an angle of about $25^{\circ}-35^{\circ}$ from the horizontal plane. The otoliths were larger in the la teral part of the macula (pars externa) than in the medial part of it (pars interna) except in both margins and the top of the otolithic layer where there were very small otoliths varying less in size. The otolithic layer was thinner and nearer to the surface of the sensory epithelium toward the periphery of the otolithic membrane. In the medial indented portion of the macula, an extremely thin otolithic layer with small otoliths was found. In the pars externa of the macula utriculi, the otolithic layer was generally thicker than in the pars interna. In the striola, the otolithic layer was generally thinner and formed a concave groove on the surface of the otolithic membrane. The otolithic layer was generally nearer to the surface of the sensory epithelium in the striola than in the other central part of the macula. Usually in the middle of the striola of a histological seztion (perpendicular to the long-axis of the striola), the otolithic layer was slightly folded or often two-times 


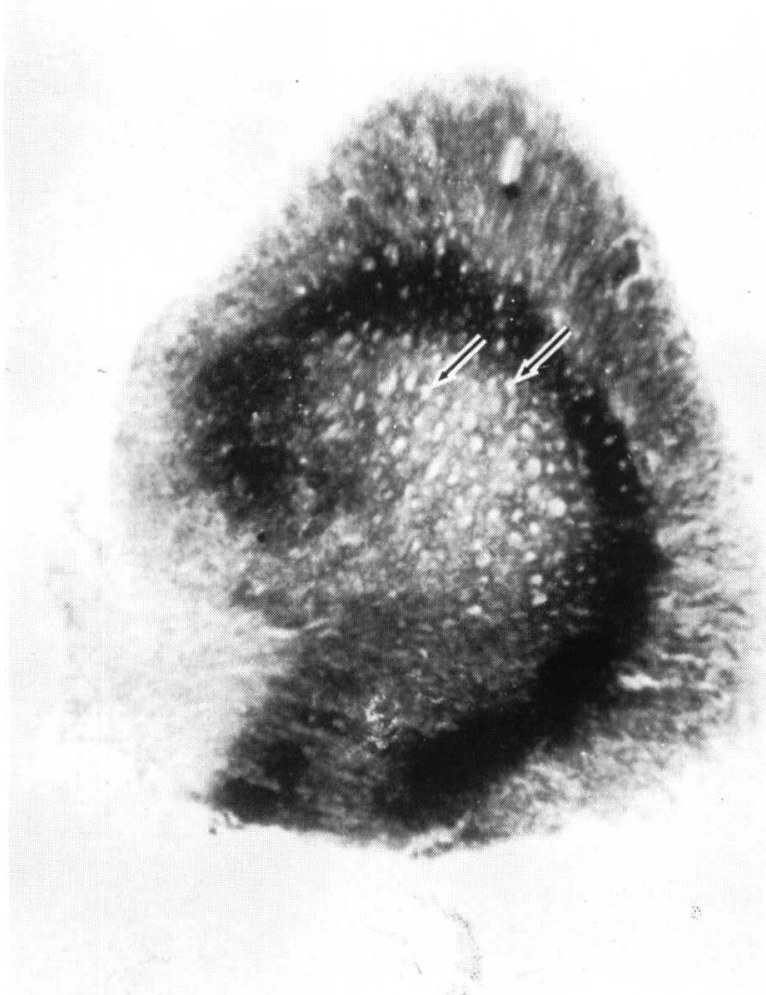

Fig. 6. Surface view of the lower surface of the macula utriculi with vertical illumination. The reticular structure of the otolithic membrane in the striola was stained darker, revealing the location of the striola. Crater-like grooves in the otolithic layer were often found (Arrow). $50 \times$.

waved and it formed otolithic elevations in a concave groove extending in the striola (Fig. 2). This folded part of the otolithic layer was more away from the surface of the sensory epithelium than the other part of the otolithic layer within the striola. Although the otolithic layer in the striola was as a whole nearer to the surface of the sensory epithelium, the lower surface of the otolithic layer in the striola formed a slight groove and it's middle section was farther from the surface. The sensory epithelium of the striola was not found to be thicker than in the other parts of the macula and no spesial epithelial elevation of the sensory epithelium was detected in the striola. In the central part of the otolithic layer, crater-like grooves were often found where the otolithic layer was extremely thin. Most of them were found in the striola and in the pars interna (Fig. 6). The upper surface of the otolithic layer showed many irregular furrows and ridges which were generally arranged radially between the striola and the peripheral margin of the macula (Fig. 4).

2) The otolithic membrane and the otoliths of the macula scaculi:

The macula sacculi was found in a vertical plane with the head up-right. The macula sacculi was a slightly concave structure extending in the resessus sphericus. The oto- 
lithic membrane covered the entire macula, which was sickle-shaped with the top of the sickle directed in the superior-posterior direction. In the striola and its extensions at both ends, the otolithic layer was thicker and formed a long series of hills on the surface of the otolithic membrane (Fig. 5). The otolithic layer was here generally nearer to the surface of the sensory epithelium than in the other central part of the macula. This central hill was usually longer than the striola itself, particularly in the main part of the macula sacculi. In the middle of the striola in a histological section (perpendicular to the longaxis of the striola), the lower surface of the otolithic layer was farther from the surface of the sensory epithelium than in both sides of the striola. Thus the surface specimen showed that the lower surface of the otolithic layer formed a slight concave groove in the striola. The upper surface of the otolithic layer showed many irregular furrows and ridges radiating from the central hill to the peripheral margin of the macula (Fig. 5). The otoliths in the lower layer were larger in the anterior-inferior part of the macula (pars externa) than in the posterior-superior part of it (pars interna). In the otolithic layer of the macula sacculi crater-like grooves, which were often found in that of the macula utriculi, were not detected in this study. On the surface of the otolithic layer of the main part of the macula sacculi, a very thin gelatin-like membrane structure was often found touching the otoliths (Fig. 2).

\section{COMMENT}

The crystalline form of calcium carbonate of the otolith differs between species of animals. The otolith crystals in fish (Sasaki ${ }^{10}$ 1970) and in amphibia (Brandenberger \& Schnitz $^{2}$ 1945, Carlström et al. ${ }^{4} 1953$, Sasaki ${ }^{10} 1970$ ) are composed of aragonite, while in man, guinea pigs, rats rabbits, birds and sharks, the crystal is calcite (Brandenberger \& Schnitz ${ }^{2}$ 1945, Carlström et al. ${ }^{4}$ 1953, Carlstrọm \& Engström ${ }^{3}$ 1955, Sasaki \& Miyata ${ }^{11}$ 1955). Several authors measured the length of an otolith crystal. It was between 1 and $20 \mu$ long in man (Carlström et al. ${ }^{4}$ 1953) and between 0.5 and $30 \mu$ long in guinea pigs (Lindeman $^{9}$ 1969), while in squirrel monkeys it was $7-8 \mu$ (Igarashi ${ }^{6}$ 1966). Recently Sasaki ${ }^{10}$ (1970) studied the otoliths of living animals, and reported that the otoliths in vivo were gel-particles under $0.1-\mu$ long, homogeneously suspended in a protein solution, which will become larger by fushing with each other after death as well as in the process of histological fixation. With regard to the giant otoliths up to $50 \mu$ long, Lindeman ${ }^{9}$ (1969) is of the opinion that they are artefacts due to a post-mortem dissolution of the crystal with subsequent re-crystallization. These artefacts were reported also by Werner ${ }^{14}$ (1940).

As far as the size of the otoliths is concerned, precise descriptions by $\operatorname{Lindeman}^{9}$ (1969) of regional differences in the otoliths were also confirmed in this study. They were, however, results obtained through study of fixed specimens of the otoliths. It is possible, therefore, that the otoliths in vivo are different. If the otoliths are uniform gel-particles in the living animal, the regional differences in the size of the otoliths constantly found in fixed specimens must be explained.

A nucleus of the otolith crystal was also found in this study (Engström ${ }^{6}$ 1951, Carl- 
ström et al. ${ }^{4} 1953$, Lindeman ${ }^{9}$ 1969). James et al. ${ }^{8}$ (1969) deterted, in the study by electron microscopy of the otoliths of 15-day-old rat embryos, rounded or irregularly shaped condensations in which a varying number of very dense granules were found, and regarded them as the first nucleation centers of calcite. It is unknown whether or not the nuclei of the otoliths are of the same nature as the primitive gel-particles of the otoliths (Sasaki $^{10}$ 1970).

With reference to the thickness of the otolithic layer on the macula sacculi, Ades and Engström $^{1}$ (1965) and Iurato ${ }^{7}$ (1967) reported that the otoliths were distributed in a snowdrift-like thickening along a curved central line corresponding to the striola region of the otolithic layer. Lindeman ${ }^{9}$ (1969) supported this finding with detailed descriptions of the distribution pattern of the otoliths in different areas. The present study is generally in agreement with Lindeman's.

In the macula scaculi, the present study confirmad also that the otolithic layer in the striola was located nearer to the sensory epithelium than that in both sides of the striola. The otolithic layer, however, was somewhat wavy in the striola with the top of the wave being farther from the sensory epithelium than the slopes and the bottom of the wave in the striola. In the macula utriculi, on the other hand, Lindeman ${ }^{9}$ (1969) reported that the lower surface of the otolithic layer in the striola was farther from the surface of the sensory epithelium than that at each side of the striola. The present study, however, showed that the lower surface of the otolithic layer in the striola was found nearer to the surface of the sensory epithelium than in each side of the striola. The wave-formation of the otolithic layer of the macula utriculi in the striola was also often detected. Thus the upper surface of the otolithic layer in the narrow middle part of the groove in the striola was usually found to be elevated, forming a snowdrift-like thickening (Fig. 4). The illustration by Lindeman ${ }^{9}(1969$, p. 34, Fig. IIa) also shows this snowdrift-like elevation of the otolithic layer within the striolar groove. It is presumable that the sensory cells in the striola are more easily stimulated by otolithic displacement than those in the other region of the macula, as the otoliths in the striola are nearer to the sensory epithelium.

With reference to the size of the otoliths in the macula utriculi, Werner ${ }^{13}$ (1933) reported that there were large otoliths in a very thick otolithic layer in the pars externa of the macula utriculi, while in the pars interna there were small otoliths in a medium-thick otolithic layer. The present study is, however, in a complete agreement with Lindeman's observation $^{9}$ (1969) that both in the maculae sacculi and utriculi there ware very large otoliths in the pars externa, medium-sized otoliths in the pars interna, and very small otoliths in the striola, in the narrow peripheral regions as well as at the top of the otolithic layer. In the present study, as was reported by Werner ${ }^{13}$ (1933), neither epithelial thickening nor epithelial elevation was noted in the striola of the sensory epithelium of both maculae. The true nature of the crater-like grooves of the otolithic layer, which were often found in the macula utriculi, is unknown. 


\section{SUMMARY}

The otolithic membrane and the otoliths were studied light-microscopically by histological sections and surface specimens of the otolithic organs of the guinea pig. The otoliths were larger in the lower portion of the otolithic layer and smaller towards it's upper surface. However, in the striola, the narrow marginal zones, and the indented portion of the macula, the ot oliths were very small and varied less in size. In the striola of the macula utriculi, the otolithic layer as a whole was thinner and it formed a concave groove on the surface of the otolithic membrane, while in the striola of the macula sacculi, the otolithic layer was thicker and formed a long series of hills. In the striola, the otolithic layer was generally nearer to the surface of the sensory epithelium than that in the other central part fo the maculae sacculi and utriculi.

\section{REFERENCES}

1) Ades, H.W., and H. ENGSTröm: Form and innervation of the vestibular epithelia. In: The role of the vestibular organs in the exploration of space, pp. 23-41. NASA SP-77, National Aeronautics and Space Administration, Washington D.C., 1965.

2) Brandenberger, E., and H.R. Schnitz: Über die Natur der Verkalkungen bei Mensch und Tier und das Verhalten der anorganischen Knochensubstanz im Falle der hauptsächlichen menschlichen Knochenkrankheiten. Helv. med. Acta, Suppl. 16, 1-63, 1945.

3) CARLSTRÖM, D., and H. Engström: The ultrastructure of statoconia. Acta oto-laryng., 45, 14-18, 1955.

4) Carlström, D., H. Engström, and S. HJorth: Electron microscopic and X-ray diffraction studies of statoconia. Laryngoscope, 63, 1052-1057, 1953.

5) Engström, H.: Microscopic anatomy of the inner ear. Acta oto-laryng., 40:5-22, 1951.

6) Igarashi, M.: Dimensional study of the vestibular end organ apparatus. In: The role of the vestibular organs in space exploration, pp. 47-53. NASA SP-115. National Aeronautics and Space Administration, Washington, D.C., 1966.

7) IUrato, S.: Submicroscopic structure of the inner ear. Pergman Press, Oxford \& London, 1967.

8) James, J., J.P.M. Schellens, and V.B. Veenhof: Electron microscopy of formation of statoconia. Experientia, 25: 1173-1174, 1969.

9) Lindeman, H.H.: Studies on the morphology of the sensory regions of the vestibular apparatus. Ergebn. Anat. u. Entwickl.-Gesch., 42; 1-113, 1959.

10) SASAKI, H.: Clinical and basic studies on the vestibular otolithic organs. Jap. Jour. Otol. Tokyo, 73: 1974-1983, 1970.

11) SASAKI, H. and J. MiYata: Experimentelle Studien uber Otolithen. Z. Laryng. Rhinol., 34: $740-748,1955$.

12) Werner, C.F.: Die Differenzierung der Maculae im Labyrinth, insbesondere bei Säugetieren. Z. Anat. u. Entwickl-Gescn., 99: 696-709, 1933.

13) Werner, C.F.: Das Labyrinth. Thieme, Leibzig, 1940.

(Received July 19, 1971) 